\title{
Compositional and frequency dependent-magnetic and microwave characteristics of indium substituted yttrium iron garnet
}

\begin{abstract}
Effect of $\operatorname{In}^{3+}$ ion substitutions on yttrium iron garnet samples' morphology, magnetic properties and dc electrical resistivity was examined closely and reported in this study. A series of polycrystalline garnet ferrites with composition of $\mathrm{Y}_{3} \mathrm{Fe}_{5-\mathrm{x}} \mathrm{In}_{\mathrm{x}} \mathrm{O}_{12}(0 \leq x \leq 0.5)$ were prepared by using the mechanical alloying technique. The morphological properties of the samples was analysed by using a TEM, XRD, FESEM and EDX. The electrical de resistivity of the samples was investigated as a function of temperature and composition by using a Picoammeter, the complex permeability was analysed by using an Impedance Materials Analyzer meanwhile microwave properties was measured by using VNA. The lattice constant increases as $\mathrm{In}^{3+}$ content increases which can be understood by the difference in ionic radii of $\mathrm{In}^{3+}$ ions replacing the smaller $\mathrm{Fe}^{3+}$ ions. The grain size also increased with $\mathrm{In}^{3+}$ content, indicating that the $\mathrm{In}^{3+}$ ion acts as a grain growth promoter. Both complex permeability components, $\mu^{\prime}$ and $\mu^{\prime \prime}$ reaching about 92.75 and 85.03 respectively at $x=0.3$; later decreased with further In substitution. This result is synchronized with FMR linewidth which manifests magnetic loss of the samples. By correlating the phase analysis, morphology, electrical resistivity and complex permeability results, it is believed that there was an increase in number of crystalline-growth regions with increasing $\mathrm{In}^{3+}$ content, which together increased a total mass of ferromagnetic grains with the latter starting to dominate the samples. The results also showed that $\operatorname{In}^{3+}$ ions increase the dc resistivity of the system.
\end{abstract}

Keyword: Dependent-magnetic; Microwave characteristics; Indium substituted; Yttrium iron garnet 